\title{
Cortical networks dynamically emerge with the interplay of slow and fast oscillations for memory of a natural scene.
}

\section{$\operatorname{AUTHOR}(\mathrm{S})$ :}

Mizuhara, Hiroaki; Sato, Naoyuki; Yamaguchi, Yoko

\section{CITATION:}

Mizuhara, Hiroaki ...[et al]. Cortical networks dynamically emerge with the interplay of slow and fast oscillations for memory of a natural scene.. Neurolmage 2015, 111: 76-84

\section{ISSUE DATE:}

2015-02-17

URL:

http://hdl.handle.net/2433/196036

\section{RIGHT:}

(C) 2015 Elsevier B.V. NOTICE: this is the author's version of a work that was accepted for publication in Neurolmage. Changes resulting from the publishing process, such as peer review, editing, corrections, structural formatting, and other quality control mechanisms may not be reflected in this document. Changes may have been made to this work since it was submitted for publication. A definitive version was subsequently published in Neurolmage, 111,2015, doi:10.1016/. neuroimage.2015.02.019; この論文は出版社版でありません。引用の際には出版社版をご確認ご利用くた さい。; This is not the published version. Please cite only the published version. 


\title{
Cortical networks dynamically emerge with the interplay of slow and fast oscillations for memory of a natural scene
}

\author{
Hiroaki Mizuhara $^{\mathrm{a}}$, Naoyuki Sato ${ }^{\mathrm{b}}$, and Yoko Yamaguchi ${ }^{\mathrm{c}}$
}

${ }^{a}$ Graduate School of Informatics, Kyoto University, Yoshida-Honmachi, Sakyo, Kyoto 606-8501, Japan. ${ }^{\text {b }}$ School of Systems Information Science, Future University Hakodate, 116-2 Kamedanakano, Hakodate, Hokkaido 041-8655, Japan. ${ }^{c}$ Neuroinformatics Japan Center, RIKEN Brain Science Institute, 2-1 Hirosawa, Wako-shi, Saitama 351-0198, Japan.

Abbreviated title: Mnemonic networks with neural oscillations

\section{Corresponding Author:}

Hiroaki Mizuhara, Ph.D.

Graduate School of Informatics, Kyoto University, Yoshida-Honmachi, Sakyo-ku, Kyoto 606-8501, JAPAN. Phone: +81-75-753-3147, FAX: +81-75-753-3147

E-mail: hmizu@i.kyoto-u.ac.jp

Conflict of Interest: The authors declare that no conflict of interest exists.

\section{Acknowledgements}

HM, NS and YY are supported by the Japanese Ministry of Education, Science, Sports and Culture (MEXT), Grant-in-Aid for Scientific Research on Innovative Areas (No.4103), 2009-2013, and HM was supported by MEXT, Grant-in-Aid for Scientific Research (A), 25240019, 2013-2017. 


\section{ABSTRACT}

Neural oscillations are crucial for revealing dynamic cortical networks and for serving as a possible mechanism of inter-cortical communication, especially in association with mnemonic function. The interplay of the slow and fast oscillations might dynamically coordinate the mnemonic cortical circuits to rehearse stored items during working memory retention. We recorded simultaneous EEG-fMRI during a working memory task involving a natural scene to verify whether the cortical networks emerge with the neural oscillations for memory of the natural scene. The slow EEG power was enhanced in association with the better accuracy of working memory retention, and accompanied cortical activities in the mnemonic circuits for the natural scene. Fast oscillation showed a phase-amplitude coupling to the slow oscillation, and its power was tightly coupled with the cortical activities for representing the visual images of natural scenes. The mnemonic cortical circuit with the slow neural oscillations would rehearse the distributed natural scene representations with the fast oscillation for working memory retention. The coincidence of the natural scene representations could be obtained by the slow oscillation phase to create a coherent whole of the natural scene in the working memory.

\section{Key words:}

Phase-amplitude coupling; Theta oscillation; Gamma oscillation; Simultaneous fMRI-EEG; Episodic memory 


\section{INTRODUCTION}

Neural oscillations are one possible mechanism of transient communication among long-distant cortices in the brain (Engel and Singer, 2001; Varela et al., 2001). Information represented in distributed cortices must be dynamically integrated into a coherent whole for appropriate function. Functional cortical networks could emerge from the neural oscillations, which take the coincidence of the spiking timings for the representation at distributed cortices (Fries et al., 2001; Fries, 2005).

The dynamic cortical networking arising from neural oscillations have often been studied in terms of the mnemonic function. The local field potential recordings in the rat hippocampus have repeatedly shown a slow oscillation in the theta frequency range as a prominent oscillatory pattern in the mnemonic function (O'Keefe and Recce, 1993; Skaggs et al., 1996; Buzsaki, 2004). The slow oscillation was also found in the human brain in association with the memory function (Kahana et al., 1999; Ekstrom et al., 2003; Mizuhara and Yamaguchi, 2011). Theoretical studies on neural oscillations have predicted the functional importance of the slow oscillation in working memory retention (Lisman and Idiart, 1995; Lisman and Jensen, 2013). The slow oscillation repeats the information coded by fast oscillation, at a specific phase for a temporal storage. Working memory can therefore be realized by the phase-amplitude coupling of the slow and fast oscillations. This phase-amplitude coupling has been repeatedly reported in the rat hippocampus in association with the mnemonic function (Tort et al., 2009; Lisman and Jensen, 2013). The interplay of the slow and fast oscillations in the human brain has also been found in ECoG recordings (Canolty et al., 2006; Axmacher et al., 
2010). Our previous study with the scalp EEG recordings also demonstrated phase-amplitude coupling of the slow and fast oscillations concerned with working memory retention of a natural scene (Mizuhara and Yamaguchi, 2011). The power of segregated fast oscillation in the gamma frequency range appeared on the frontal and parietal sites and was enhanced at a phase of the slow oscillation in the delta/theta frequency range around the frontal midline site, suggesting that a dynamic network linking of distributed cortical areas emerged with the interplay of the slow and fast oscillations for the temporal storage of the natural scene. The results were derived from the scalp EEG. Cortical regions concerning with the slow and fast oscillations are difficult to identify only from the scalp EEG measurements, because of the blur effect caused by the volume conduction. It is still an open question what the slow and fast oscillations represent in cortices.

The slow oscillation in the delta/theta frequency range may involve mnemonic circuits, while the fast oscillations in the gamma frequency range are items themselves that are retained in the working memory for a while, if the slow oscillation rehearse the information coded by the fast oscillation at a specific phase for temporal memory storage. The retrieval and rehearsal of items could realize the working memory along with the interplay of the slow and fast oscillations. We aimed to verify our hypothesis by recording EEG and functional magnetic resonance imaging (fMRI) simultaneously during working memory retention of natural scenes. This simultaneous EEG-fMRI recording enables us to identify the cortical networks involved with the slow and fast oscillations. Natural scenes are known to activate the parahippocampal place area in the human medial temporal cortex (Epstein and Kanwisher, 1998). The cortical networks concerned with neural 
oscillations would thus include the medial temporal cortex, along with distributed cortices for the mnemonic function of natural scenes.

The combination of the attentional blink procedure and delayed match to sample task was used in order to actively manipulate participants' behavioral performance of working memory retention. The first and second targets in a rapid serial visual presentation (RSVP) are digit numbers and natural scenes. The attentional blink procedure can modulate the working memory performance restrictively for the natural scene by varying the stimulus onset asynchrony of the first and second targets (SOA), while the working memory performance for the digit numbers still remain. The fMRI analysis confirms whether the working memory retention for the natural scene is selectively manipulated by the attentional blink procedure. The comparison between SOA conditions was used to identify the activation in cortical areas for scene processing. The EEG analysis confirms the phase-amplitude coupling of the slow and fast oscillations in association with the working memory retention. The combined analysis of simultaneously recorded EEG-fMRI was then applied to identify cortical regions correlated with the temporal fluctuation of EEG power. These analyses enable to show the functional relevance of the slow oscillation to the mnemonic circuit and the fast oscillation to the perceptual representations. 


\section{MATERIALS \& METHODS}

Participants. Twenty healthy males, aged 21 to 30 years old (mean \pm s.d.: $25.6 \pm 3.3$ years old) and all right-handed (Edinburgh handedness test, mean \pm s.d.: $96.5 \pm 7.5$ ), participated in the experiments. Six participants were excluded from further analyses because of low behavioral accuracy in recognition memory ([accuracy for first target] $<90 \% \cap$ [accuracy for second target $]_{\mathrm{SOA}=800 \mathrm{~ms}}<40 \%$ ). One participant was excluded from the EEG analysis and combined EEG and fMRI analysis because of excessive artifacts that could not be removed by EEG preprocessing. The experimental procedure was approved by the ethics committee at the Graduate School of Medicine and the Faculty of Medicine, Kyoto University (\# E311). Participants gave their written informed consent according to the Declaration of Helsinki and were paid for their participation in the study.

Task Procedure. The task was similar to the task described in our previous study (Mizuhara and Yamaguchi, 2011). An attentional blink procedure was combined with a delayed match to sample task to actively manipulate participants' performance of working memory retention of a natural scene. The attentional blink procedure can manipulate the perceptual awareness of a secondary presented stimulus by embedding the two stimuli with a short SOA into a RSVP (Fig. 1A). In the attentional blink procedure, we used four-digit numbers as the first targets (Fig. 1B) and natural scenes as the second targets (Fig. 1C), as in our previous study (Mizuhara and Yamaguchi, 2011). Both the first and second targets appeared in an RSVP stream. The participants were required to memorize both targets. Each target and distracter was presented for $100 \mathrm{~ms}(10$ 
$\mathrm{Hz}$ ), with SOA was set at 300 or $800 \mathrm{~ms}$. The first target was presented at the third, fourth, or fifth presentation position of the RSVP stream. After the second target presentation, nine distracters were presented in the RSVP stream. The distracters were made from the natural scenes used as the second target. The scenes were first divided into 192 (horizontal $\mathrm{x}$ vertical $=16 \times 12$ ) square blocks, and their positions were then randomly scrambled for the distracters. Black grids were drawn on the boundaries of the blocks over the scrambled images. The grids were also drawn on the same positions of the target natural scenes. The second target images and distracter images subtended approximately $10^{\circ} \times 8^{\circ}$. The delay period of $9 \mathrm{~s}$ was embedded after the end of the RSVP stream. This delay period was defined as the 'retention interval' for the working memory. A fixation was presented at the center of a white image during the retention interval. Probe stimuli for the first and second target were presented for $1.5 \mathrm{~s}$ after this interval. Participants were required to indicate whether the probe stimulus 'was present' in the stream, 'was not present' in the stream, or 'cannot be judged' by pressing one of three keys as accurately and rapidly as possible. Half of the probe stimuli were 'matches' and half were 'non-matches' for each SOA. The next RSVP stream began $1 \mathrm{~s}$ after the end of the second target probe presentation. All SOA conditions and 'match/non-match' conditions were presented in pseudo-random order. The experiments consisted of 3 sessions for a participant, and the session consisted of 28 trials (i.e., 28 RSVP streams).

[Figure 1 about here]

Simultaneous Recording of fMRI and EEG. Two MR compatible amplifiers (Brain Vision MR, 
Brain Products, Germany) were used to acquire the EEGs during fMRI measurements. We used a $10 \%$ standard system electrode cap with sintered $\mathrm{Ag} / \mathrm{AgCl}$ ring electrodes (Brain Cap, Falk Minow Services, Germany). The electrodes had 62 EEG channels, one electrocardiogram (ECG) channel, and one electrooculogram (EOG) channel. An $\mathrm{FCz}$ electrode, located between $\mathrm{Fz}$ and $\mathrm{Cz}$, was used as the measurement reference, while an electrode on the inion was used as the measurement ground. The ECG electrode was set at the back and the EOG electrode was set below the eye at the face. The Brain Vision Recorder (Brain Products, Germany) was used to sample the raw EEG at $5 \mathrm{kHz}$ with $1 \mathrm{~Hz}$ high pass and 250 $\mathrm{Hz}$ low pass filters with a notch filter $(60 \mathrm{~Hz})$. The power line frequency at the experimental site was $60 \mathrm{~Hz}$. Blood oxygenation sensitive echoplanar images (EPI), obtained using a 1.5 T MR scanner (Magnex Eclipse, Shimadzu-Marconi), were taken simultaneously with the scalp EEG measurements. The fMRI measurement conditions were as follows: repetition time $=4 \mathrm{~s}$, acquisition time $=3 \mathrm{~s}$, echo time $=50 \mathrm{~ms}$, flip angle $=90$ deg., field of view $=192 \mathrm{~mm}$, in-plane resolution $=64 \times 64,30$ axial slices, and slice thickness $=4 \mathrm{~mm}$ with a $1 \mathrm{~mm}$ gap (descending slice order). The MRI measurement was the sparse sampling with $3 \mathrm{~s}$ scanning and $1 \mathrm{~s}$ inter-scan interval to subsequently identify the onsets of MR artifacts. In total, 108 volumes were acquired in one session over $7 \mathrm{~min}$. The first three volumes were discarded from the subsequent analysis. The participant's head was immobilized by a vacuum pillow during the measurements.

fMRI Analysis. We used SPM8 software (Wellcome Department of Cognitive Neurology, London, UK, URL: www.fil.ion.ucl.ac.uk/spm) for image preprocessing and voxel-based statistical analysis. The slice 
timing was corrected with respect to the middle slice to remove the time delay of scanning the entire brain. The remaining EPIs (105 volumes for each session $\times 3$ sessions) were transferred into the first image volume for each participant to correct for head motion. The individual EPIs were normalized to a standard brain by applying the parameters estimated by matching the $\mathrm{T} 1$ anatomical image to the stereotactic coordinate image from the Montreal Neurological Institute. The EPIs were then smoothed with an $8 \mathrm{~mm}$ full-width half-maximum Gaussian kernel. A voxel-based statistical analysis was performed on the preprocessed EPIs using the general linear model. We compared the fMRI BOLD responses between the conditions of SOA=300 $\mathrm{ms}$ and $\mathrm{SOA}=800 \mathrm{~ms}$ to identify the cortical areas associated with the modulation of working memory accuracy. The starting point for the retention interval for the $\mathrm{SOA}=300 \mathrm{~ms}$ and $\mathrm{SOA}=800 \mathrm{~ms}$ conditions (Fig. 1a) were respectively modeled as the onsets of two box-car functions. The duration was set at $9 \mathrm{~s}$ from the onsets; i.e., the duration of the retention intervals. The box-car functions were convolved with the canonical hemodynamic response function, and then used as the regressors for the regression analysis. The six head motion parameters, derived from the realignment processing, were also used as regressors to reduce the motion related artifacts. Before computing the regression analysis, the low-frequency confounding effects were removed using a high-pass filter with a $120 \mathrm{~s}$ cutoff period, and serial correlations among scans were estimated with an autoregressive model $[\mathrm{AR}(1)]$ to remove the high-frequency noise that contaminated in the EPI time series. The regression coefficients for the retention interval were computed for each individual using the fixed-effect model and then taken into the group analysis using a random-effect model of a paired t-test 
between the conditions of $\mathrm{SOA}=300 \mathrm{~ms}$ and $\mathrm{SOA}=800 \mathrm{~ms}$.

EEG Analysis. MR imaging and cardioballistic artifacts contaminated the EEG measurements simultaneously measured with fMRI. The artifacts were removed by the following analysis using Brain Vision Analyzer software (Brain Products, Germany). The measured EEG data were first up-sampled from $5 \mathrm{kHz}$ to $50 \mathrm{kHz}$ with spline interpolation to identify the exact timing of the onsets of the MR scan. The onsets of the MR scan were identified by the temporal difference in the EEG amplitude time series. The averaged waveforms of the MR scan artifacts, computed based on the onsets of the MR scans, were subtracted from the contaminated periods (Allen et al., 2000). The EEGs were then low-pass filtered with $100 \mathrm{~Hz}$ cutoff frequency and down-sampled to $500 \mathrm{~Hz}$. Next, the R peaks of the ECG were identified by eye inspection with a semi-automatic support for marking up the peaks based on the coherence of ECG waveform to the template ECG waveform. The cardioballistic artifacts were then removed by subtracting the artifacts waveform, which was computed by averaging based on the R peaks (Allen et al., 1998). Ocular artifacts in the EEG were also corrected (Gratton et al., 1983) by subtracting the voltages of the VEOG and HEOG channels, multiplied by a channel-dependent correction factor calculated on the basis of linear regression among VEOG/HEOG and EEG electrodes. Here, VEOG and HEOG were computed by subtracting the EOG data from EEG at the Fp1 and Fp2 electrodes. The purpose of this study was to identify cortical areas concerned with the EEG components. A combined analysis requires the use of a continuous time series of EEG power in the regression analysis between the EEG power fluctuation and BOLD time series (these details are described later). We 
therefore decided to use all trials, without discarding any, for the EEG analysis. Note that a risk arises for artifact contamination with the use of whole data without any selection. We used independent component analysis (ICA) to reduce the risk of the artifact contamination into the EEG data. The ICA was applied to the EEG data to identify and remove the remaining ECG artifacts and other artifacts. The EEG data were decomposed into 62 independent components by ICA. Those ICA components with remaining ECG artifacts were identified by the Brain Vision Analyzer software. This excluded $8.9 \pm 1.4 \%$ (mean \pm standard deviation across participants and sessions) of the ICA components from the back transformation of the ICA component matrix to the EEG data. The reference was then changed to the average of all scalp electrodes except the VEOG and HEOG. The preprocessed EEG data were exported to Matlab (Mathworks Inc., USA) software for further analysis.

We identified the EEG activity involved in the working memory retention by comparing the EEG power between the conditions of $\mathrm{SOA}=800 \mathrm{~ms}$ and $\mathrm{SOA}=300 \mathrm{~ms}$. Prior to the comparison, we used ICA to de-noise the EEG data (Scheeringa et al., 2011). We concentrated on the EEG activity around $3 \mathrm{~Hz}$, since our previous scalp EEG study showed EEG activity enhancement at $3 \mathrm{~Hz}$ around the central sites. The low frequency EEG component was extracted by applying a 2.0-4.0 Hz band-pass filter to the preprocessed EEG data prior to the ICA decomposition. The filtered data were z-transformed and whitened as the preprocessing for the ICA analysis. The ICA decomposition was performed with the fast ICA as follows (Hyvarinen and Oja, 2000). The unmixed weights of ICA analysis were obtained by applying the fast ICA to the whitened EEG 
data. The derivative function of a nonquadratic nonlinearity was defined as 'tanh' in the fast ICA, and all the independent components were estimated in parallel. Application of the band-pass filter to the EEG data revealed that some activities showed monotonic increases or decreases within the band-pass frequency range. These activities were the extension of the activities that had peaks outside the frequency range. A few independent components, which showed the EEG power peak around the central electrodes sites at $\sim 3 \mathrm{~Hz}$, were thus chosen by eye inspection $(1.9 \pm 0.6$ components $)$. We applied the unmixed weights of these independent components to the unfiltered EEG data.

The EEG data derived from the ICA decomposition were transformed into a time-frequency representation of EEG power from $1 \mathrm{~Hz}$ to $100 \mathrm{~Hz}$, in 40 logarithmically spaced steps, using the Morlet wavelet. The time-frequency representation of the EEG power was computed by the wavelet transformation, whose mother wavelet is defined by $w(t, f)=\left(\sigma_{t} \sqrt{\pi}\right)^{-\frac{1}{2}} \exp \left(-t^{2} / 2 \sigma_{t}^{2}\right) \exp (i 2 \pi f t)$, where $t$ is time, $f$ is frequency and $\sigma_{f}$ is the variance at the frequency $f$ (Tallon-Baudry et al., 1997). The wavelet is characterized by the ratio of the frequency to the variance $\left(f / \sigma_{f}\right)$, the so-called 'width' in the wavelet analysis, and was set to be $f / \sigma_{f}=10$. The mother wavelet was convolved to the EEG time series $S(t)$ and the EEG power $E(t, f)$ at electrode $n$ was computed by $E(t, f)=\log _{10}|w(t, f) \otimes S(t)|^{2}$, where || denotes the absolute value, and $\otimes$ is the convolution. The phase of slow oscillation $(\phi(t, f))$ was computed by $\exp (i \phi(t, f))=w(t, f) \otimes S(t) /|w(t, f) \otimes S(t)|$. We then compared the EEG power between the conditions of $\mathrm{SOA}=300 \mathrm{~ms}$ and $\mathrm{SOA}=800 \mathrm{~ms}$ by a paired $\mathrm{t}$-test. The multiple comparison was corrected by a nonparametric 
statistical test (Maris and Oostenveld, 2007). In this method, an empirical t-value was estimated by a permutation-based Monte Carlo approach. Briefly, the two conditions were collected into a single set, one half was then randomly chosen and compared with the rest by the t-test. This process was repeated 10,000 times, and a histogram was constructed. A cluster algorithm-based approach was then used to correct the multiple comparison. The adjacent time and frequency samples were classified into a connected set. We then calculated the cluster-level statistics by taking the mean of the EEG power within the cluster, and compared it between the conditions. The threshold for the cluster-level statistics was decided by the histogram derived from the permutation-based Monte Carlo approach (corrected $\mathrm{p}<0.05$ ).

The phase-amplitude coupling was evaluated by the modulation index (Canolty et al., 2006; Roux et al., 2013), defined by $\mathrm{MI}=\left|\sum z(t) / N\right|$, where $N$ is the total time points of the retention interval. The complex variable $z(t)$ was computed by $z(t)=a\left(t, f_{\text {fast }}\right) \exp \left(i \phi\left(t, f_{\text {slow }}\right)\right)$, where $a\left(t, f_{\text {fast }}\right)$ denotes the analytic amplitude of fast oscillations computed by wavelet transformation. The analytic amplitude was obtained from the raw EEG data without ICA de-noising. Before applying it to the computation of modulation index, the analytic amplitude was z-transformed by demeaning and divided by the standard deviation. The statistical significance of the modulation index was evaluated by comparison to the surrogate data. The amplitude for the surrogate data was derived by randomly shifting the phase in time (Roux et al., 2013). The complex variable consisted of the time-shifted phase and the analytic amplitude. We then computed the modulation index. This procedure was repeated 200 times to construct an empirical distribution of the 
surrogate data, and the median of the distribution was used as the surrogate data for each participant, phase, and power. The multiple comparison was corrected by the permutation-based Monte Carlo approach in the same manner as the EEG power analysis. The cluster was defined as the adjacent electrode samples in this analysis. The threshold for the cluster-level statistics was $p<0.001$ (corrected).

Combined EEG-fMRI analysis. The cortical regions concerned with the slow and fast EEG oscillations were identified by computing the correlation of the BOLD time series to the EEG power time series. The signal was derived from the ICA of EEG. A time series of slow EEG power was computed by averaging across dominant frequency range for the better accuracy of working memory retention $(1-4 \mathrm{~Hz})$, and the independent components $(1.9 \pm 0.6$ components). The convolution of the EEG power time series and the canonical HRF was computed to model the BOLD changes of cortical regions. The EEG based BOLD signal was used as an explanatory variable in the regression analysis of fMRI. The fast EEG power was also used as a regressor instead of the slow EEG power for identifying the cortical areas concerned with the fast oscillations. The time series for the fast oscillation was derived from the wavelet transformation of the raw EEG data without ICA de-noising. Note that the preprocessing differed slightly from that for the slow oscillation. The ICA components were used as the regressors for the slow oscillation based on an a priori assumption about the slow oscillation derived from our previous study (Mizuhara and Yamaguchi, 2011). In the simultaneous EEG-fMRI, the frequency of the ECG artifacts neighbors the frequency of the slow oscillation. We thus applied the ICA to the EEG data for a careful selection of the intended oscillation, and 
computed the hierarchical coupling of the fast EEG power to the phase of this EEG component. The purpose of the simultaneous EEG-fMRI was to identify cortices that correlated with the EEG activities showing the phase-amplitude coupling. In the phase-amplitude coupling analysis, we used the raw EEG data for computing fast oscillation power and the ICA components for computing the slow oscillation phase. We therefore used the raw EEG data as a regressor for the fast oscillation, instead of the ICA components for the slow oscillation. The EEG power time series was averaged across $30 \sim 100 \mathrm{~Hz}$ and all scalp electrodes, and convolved with the HRF. The EEG activity in the higher frequency range showed a clear MI difference for the $\mathrm{SOA}=800 \mathrm{~ms}$ condition compared to the surrogate data (see Results), thereby identifying cortical areas that showed phase-amplitude coupling to the slow oscillation.

Note that the slow EEG components used for the regressor had an EEG power difference between the condition of $\mathrm{SOA}=800 \mathrm{~ms}$ and $\mathrm{SOA}=300 \mathrm{~ms}$. It may cause an artifactual correlation in the fMRI results (slow oscillation: $r=-0.13 \pm 0.02$ for $\mathrm{SOA}=300 \mathrm{~ms}$ condition, $r=0.02 \pm 0.02$ for $\mathrm{SOA}=800 \mathrm{~ms}$ condition; fast oscillation: $r=-0.04 \pm 0.02$ for $\mathrm{SOA}=300 \mathrm{~ms}$ condition, $\mathrm{r}=-0.07 \pm 0.02$ for $\mathrm{SOA}=800 \mathrm{~ms}$ condition). We excluded this risk by modeling a time series by convolving the HRF and the retention interval for condition of $\mathrm{SOA}=300 \mathrm{~ms}$ and $\mathrm{SOA}=800 \mathrm{~ms}$, which were used in the conventional fMRI analysis. This time series was also included in the regression analysis as explanatory variables. The timing of the response button presses was also included in the explanatory variables to reduce the risk that the cortical regions merely reflected the motor activity for the responses. The six head motion parameters, derived from the realignment processing, 
were also used as additional regressors to reduce the motion related artifacts.

The low-frequency confounding effects were removed before computing the regression analysis by using a high-pass filter with a $120 \mathrm{~s}$ cutoff period, and serial correlations among scans were estimated with an autoregressive model (AR(1)) to remove the high-frequency noise that contaminated the EPI time series. The regression coefficients for the EEG power time series were computed for each individual by using the fixed-effect model and then taking each into the group analysis using a random-effect model of a one sample t-test.

This combined EEG-fMRI analysis was derived from the regression analysis by using the EEG power fluctuation as an explanatory variable. We used the EEG power time series for the slow and fast oscillation as the explanatory variable. Note that the slow and fast oscillations showed hierarchical coupling in the phase-amplitude coupling analysis. This may indicate a correlation between the slow and fast EEG power time series. Therefore, both slow and fast EEG power time series were included in the regressors in the additional analysis to confirm whether the cortices derived from that analysis were concerned with slow or fast oscillations. This inclusion enabled us to separately identify cortices for the slow and fast oscillations. In this analysis, a task-design derived regressor, button press timing, and six head motion parameters were also included in the explanatory variables. 


\section{RESULTS}

\section{Behavioral Results}

A significant manipulation of the accuracy of the second target retention by the attentional blink procedure was confirmed by comparing the accuracies of working memory retention between conditions of $\mathrm{SOA}=300 \mathrm{~ms}$ and $\mathrm{SOA}=800 \mathrm{~ms}$ (Fig. 2). The analysis of variance (ANOVA) showed a significant main effect of the accuracy in recognition memory on the factor for the first and second target retention conditions $[F(1,13)=466.0, p<0.001]$ and the interaction $[F(1,13)=10.6, p=0.006]$, while no significant main effect was seen on the factor for the SOA conditions $[\mathrm{F}(1,13)=4.0, \mathrm{p}=0.07]$. The post-hoc analysis demonstrated a simple main effect of the SOA conditions for the second target $[\mathrm{F}(1,26)=12.0, \mathrm{p}=0.002]$, while no significant difference was seen for SOA for the first target $[\mathrm{F}(1,26)=0.02, \mathrm{p}=0.88]$. Note that the participants appropriately engaged in the experiments, while the accuracy for the second target was somewhat low. The task was to judge whether the probe stimulus was presented in the RSVP stream, and the participants were required to reply with one of three choices (present/absent/ambiguous). The resulting chance level was 33\%. The one sample t-test confirmed that the accuracy for T2 was significantly greater than the chance level $[\mathrm{SOA}=300 \mathrm{~ms}: \mathrm{t}(13)=5.72, \mathrm{p}<0.001 ; \mathrm{SOA}=800 \mathrm{~ms}: \mathrm{t}(13)=8.34, \mathrm{p}<0.001]$. The rate for choosing the 'ambiguous' option was $31.1 \pm 5.1 \%$. This rate was not sufficiently high to say the participants did not engage in the task. The low recognition accuracy might be derived from the short presentation time of the natural scenes. The duration of the natural scene presentation was $100 \mathrm{~ms}$. This might be too short to fully recognize 
the natural scene. Note that the difference in the recognition accuracy was a fair reflection of the difference in the performance of the working memory retention, not merely the difference in the confidence level between the conditions. The rate for choosing the 'ambiguous' option did not show a significant difference between the conditions (SOA main effect: $\mathrm{F}(1,13)=3.814, \mathrm{p}=0.073$; target main effect: $\mathrm{F}(1,13)=38.9, \mathrm{p}<0.0001$; SOA $\times$ target interaction: $\mathrm{F}(1.13)=3.197, \mathrm{p}=0.097)$. The results confirmed that the attentional blink procedure was able to manipulate the working memory performance of only the natural scene.

[Figure 2 about here]

\section{fMRI Results}

In the current study, we used a natural scene as the second target. A natural scene can activate the parahippocampal place area in the medial temporal cortex (Epstein and Kanwisher, 1998; Epstein, 2008). The behavioral results showed a difference in accuracy of natural scene memory between the conditions of $\mathrm{SOA}=300 \mathrm{~ms}$ and $\mathrm{SOA}=800 \mathrm{~ms}$. The natural scenes were maintained for the condition of $\mathrm{SOA}=800 \mathrm{~ms}$, but poorly maintained for the condition of $\mathrm{SOA}=300 \mathrm{~ms}$. This should lead the relatively larger activation in the parahippocampal place area for the condition of $\mathrm{SOA}=800 \mathrm{~ms}$ than for $\mathrm{SOA}=300 \mathrm{~ms}$. As expected, the BOLD in the right parahippocampal place area for the condition of $\mathrm{SOA}=800 \mathrm{~ms}$ showed a larger response than that for the condition of $\mathrm{SOA}=300 \mathrm{~ms}$ (cluster-level FDR corrected $\mathrm{p}<0.05$; Fig. 3, Table 1). This showed that greater activity in the parahippocampal place area is concerned with better accuracy for memory retention of the natural scene. The other significant increases in the BOLD signals during the retention interval occurred in 
the bilateral fusiform cortices and right occipital cortex for the condition of SOA=800ms. No significant increase in the BOLD signal was found for the condition of SOA $=300 \mathrm{~ms}$.

[Figure 3 about here]

\section{EEG Results}

A slow oscillation around $3 \mathrm{~Hz}$ at the central electrode sites showed the EEG power enhancement in association with the better accuracy of memory retention in a similar task as used in the current study and the scalp EEG recording, without fMRI measurement (Mizuhara and Yamaguchi, 2011). The comparison of the EEG power of the retention interval between the conditions of $\mathrm{SOA}=800 \mathrm{~ms}$ and $\mathrm{SOA}=300 \mathrm{~ms}$ confirmed the existence of the slow EEG oscillation around 3Hz (Fig. 4A). The EEG activity clearly appeared at the frontal midline electrodes. This frequency and spatial topography of the slow EEG activity is consistent with our previous scalp EEG study (Mizuhara and Yamaguchi, 2011).

The phase of slow oscillation was coupled with the amplitude of the higher gamma oscillation in our previous study (Mizuhara and Yamaguchi, 2011). The current results confirmed that the slow oscillation showed a clear phase-amplitude coupling to the fast EEG oscillation around $50 \mathrm{~Hz}$ for the successful memory retention (condition of $\mathrm{SOA}=800 \mathrm{~ms}$ ) (Fig. 4B). Note that results are shown only for the $\mathrm{SOA}=800 \mathrm{~ms}$ condition. In the EEG power comparison, the slow EEG power around $3 \mathrm{~Hz}$ for the $\mathrm{SOA}=800 \mathrm{~ms}$ condition was relatively larger than that for the $\mathrm{SOA}=300 \mathrm{~ms}$ condition. The difference in the EEG power could result in the difference in the signal-to-noise ratio of the phase computation between the conditions. We did not 
compare the modulation indices between the conditions. The result for the $\mathrm{SOA}=800 \mathrm{~ms}$ condition agreed well with our previous study, where the power of higher gamma activity showed hierarchical coupling to the phase of $3 \mathrm{~Hz}$ slow oscillation of the frontal electrode (Mizuhara and Yamaguchi, 2011).

[Figure 4 about here]

\section{EEG correlated fMRI Results}

Cortical regions for the slow oscillation were identified to verify whether the slow oscillation was associated with a mnemonic circuit for a natural scene. When the slow oscillation appeared at the central electrodes site, the retrosplenial cortex were activated (cluster-level FDR corrected $\mathrm{p}<0.05$, Fig. 5, Table 2). The cortex is known as a brain region for the mnemonic function. The activity was also found in the supplementary motor cortex, the left parahippocampal place area/fusiform cortex, and the left occipital cortex. These were the regions where the BOLD signal increased when the slow EEG power was enhanced. The fast EEG power also showed a positive correlation with the BOLD in the left parahippocampal place areas, and the occipital/fusiform cortices. Note that the slow oscillation showed hierarchical coupling to the power of the fast EEG oscillation (Fig. 4). The correlation of the slow and fast EEG power $(r=0.19 \pm 0.02, p=0.03 \pm 0.02)$ could cause an overlapped observation for the results for both the slow and fast oscillations. We confirmed which of these cortical areas is more concerned with either the slow or fast oscillations by the inclusion of both the slow and fast EEG power time series in the regression analysis. The parahippocampal place area and the occipital/fusiform cortex appeared to be more tightly coupled with the fast oscillation (unc. $p<0.001$ for 
voxel-level multiple comparison) than with the slow oscillation. The retrosplenial cortex showed a tighter coupling with the slow EEG power (unc. $\mathrm{p}<0.001$ for voxel-level multiple comparison). The supplemental motor cortex did not exceed the same statistical threshold as these activities for either the slow or fast oscillations in this analysis. The activity in the supplementary motor cortex was therefore discarded in further discussion. The BOLD signal showed no decrease associated with the slow and fast EEG power enhancement.

The combined EEG-fMRI analysis showed that small head motions may result in an artifactual correlation of the EEG power time series with the BOLD. An abrupt head motion showed a correlation with the activity in the spontaneous motion related cortices (Jansen et al., 2012). These motions are sometimes correlated with the EEG power regressions in the combined EEG-fMRI analysis. Before applying the combined EEG-fMRI analysis in the current study, we used the ICA to detect only the slow components in the theta frequency range, and used this as the explanatory variable in the regression analysis. We selected only a few components based on a priori knowledge from our previous study on the pure EEG recording (Mizuhara and Yamaguchi, 2011).

The use of the ICA could denoise the motion-related EEG activity; however, the results still have a risk of motion-related activity. We confirmed that the slow EEG power was not merely the by-product of a small head motion by computing the correlation between the EEG power regressors and the small head motion-related activity. The head motion was detected by the method proposed by Jansen et al. (2012). Briefly, the first derivative of z-scored six realignment parameters of fMRI was computed and summed. The time 
series was z-scored again. The time course exceeding 1 created a single time series of delta function indicating the head movement. The canonical HRF was convolved with this time series to create an abrupt movement regressor. We then computed the correlation coefficients between the movement regressor and EEG power regressors and found no correlation between them (slow EEG: $r=0.013 \pm 0.022$; fast EEG: $\mathrm{r}=0.016 \pm 0.024$ ). The mean correlation coefficients among participants did not differ from zero (slow EEG: $\mathrm{t}(12)=0.61, \mathrm{p}=0.55$; fast EEG: $\mathrm{t}(12)=0.69, \mathrm{p}=0.50$ ), which implied that no correlation existed between the EEG regressors and the movement regressors in the combined EEG-fMRI analysis.

[Figure 5 about here] 


\section{DISCUSSION}

The enhancement of low frequency oscillation was reported as important EEG activity in the memory retention of natural scenes (Mizuhara and Yamaguchi, 2011). The current results were consistent with the previous study. The slow oscillation around the delta/theta frequency band appeared in association with the better accuracy of memory retention (Fig. 4A). The combined analysis of simultaneous EEG-fMRI identified cortices where the fMRI signals were correlated with the temporal fluctuation of the slow oscillation EEG power: the retrosplenial cortex (Fig. 5) is a well-known cortex in scene processing (Epstein, 2008). The temporal fluctuation of the slow oscillation EEG power also showed a correlation with the high frequency oscillation (Mizuhara and Yamaguchi, 2011). The slow and fast oscillations showed the phase-amplitude coupling for successful memory retention. The current results replicated the findings of the relationship between the slow and fast oscillations (Fig. 4B). The combined analysis of simultaneous EEG-fMRI showed a correlation between the fast EEG power and the activity in the parahippocampal place area (Fig. 5). The parahippocampal place area is a crucial cortex for natural scene processing (Epstein and Kanwisher, 1998; Epstein, 2008). The occipital/fusiform cortex was also the area where the fMRI signal was coupled with the fast oscillation.

In the current study, we used a combination of the attentional blink procedure and delayed match to sample task to investigate working memory retention. The mechanism of the attentional blink effect is still an open question; however, this effect is widely accepted to arise from a failure in the deployment of the 
attentional resource at the stage of visual working memory. A functional brain imaging study showed that the interaction between the attention and working memory systems (Johnston et al., 2012) could concern either the encoding of the visual information into the working memory, or the retrieval of the information from the memory. The use of a combination of the attentional blink procedure and delayed match to sample task may create difficulty when attempting to discuss whether the observed effect in the current study is concerned with the interaction of the attention and working memory systems, or purely with working memory retention. We assumed that the attentional blink effect must have occurred at the time of the second target presentation. The typical attentional blink effect mainly appears $300 \mathrm{~ms}$ after the first task presentation, and lasts only a few hundred milliseconds (Johnston et al., 2012). The retention interval in the current attentional blink task procedure was sufficiently long to avoid the first task effect. In the current study, we used the interval from the termination of RSVP stream to the onset of the prove stimulus presentation for the EEG and combined EEG-fMRI analyses. The termination of the RSVP stream was $1 \mathrm{~s}$ after the second target presentation onset, and the retention interval was $9 \mathrm{~s}$. The majority of the retention interval was therefore sufficiently far from the first and second target presentations. No first target effect would occur on the working memory during the retention interval. We were therefore able to treat the working memory 'maintenance' solely, rather than the manipulation (retrieval/encoding) of the information in the memory. Another risk with this task arose when the items were lost from their participant's recognition memory. We assumed that the attentional blink would distract the encodings of the items into the memory. The recognition performance, however, may be affected 
by forgetting items in the working memory during the retention interval. Note that the task design was identical during the retention interval between the conditions. The effect on the behavioral performance would be same concerning the forgotten items during the retention interval, although we cannot completely isolate the effect on the recognition memory performance caused either by the impairment of the encoding or the retention. The difference in the recognition accuracy would come from the manipulation of the encodings of the items into the working memory by the attentional blink procedure.

\section{Retrosplenial activity with slow oscillation}

The retrosplenial cortex is a crucial node of the cortical network for processing of natural scenes (Epstein, 2008). Lesion studies of the retrosplenial cortex in humans showed difficulties in navigation (Bottini et al., 1990; Suzuki et al., 1998). The impairment of navigation was also found in rats with lesions of the retrosplenial cortex (Aggleton and Vann, 2004). These studies on the deficits support the functional importance of the retrosplenial cortex for the scene processing. The retrosplenial cortex can be activated by scene viewing and also by scene imagery (O'Craven and Kanwisher, 2000; Ino et al., 2002). The current results were derived from the retention interval where the scene was not presented to the participants. The activity in the retrosplenial cortex was identified as correlating with the slow EEG power, which was enhanced during this interval for working memory retention (Fig. 5). The activity was a response to the scene imagery during the retention interval.

The retrosplenial cortex is strongly interconnected with the parahippocampal place area (Kobayashi 
and Amaral, 2000), which is another key node of the cortical network for scene processing. The activity in the parahippocampal place areas was identified as showing a correlation with the fast EEG power fluctuation. The scene imagery might be achieved by the interplay of the retrosplenial cortex in the slow oscillation and the parahippocampal place area in the fast oscillation during the retention interval.

\section{Phase-amplitude coupling of slow and fast oscillations}

The slow oscillation phase and fast oscillation power showed phase-amplitude coupling during the retention interval (Fig. 4B). Previous theoretical studies predicted the functional importance of phase-amplitude coupling (Lisman and Idiart, 1995; Jensen and Colgin, 2007; Lisman and Jensen, 2013). Information coded by the fast oscillation is considered to be rehearsed at an ideal phase of the slow oscillation for working memory retention. Experimental evidence was provided in support of this theory. An ECoG study showed clear phase-amplitude coupling of the slow and fast oscillations during cognitive tasks including working memory retention (Canolty et al., 2006). The strength of the phase-amplitude coupling of ECoG was correlated with the working memory load in the human hippocampus (Axmacher et al., 2010). The slow and fast oscillations seen on the scalp EEG in our previous study also showed phase-amplitude coupling for the memory retention of a natural scene, where the frontal and parietal gamma activities were simultaneously enhanced at a phase of frontal slow oscillation (Mizuhara and Yamaguchi, 2011). The current result also followed the findings of the phase-amplitude coupling (Fig. 4B).

The slow oscillation showed a temporal correlation to the activity in the retrosplenial cortex in the 
combined analysis of simultaneous EEG-fMRI (Fig. 5). The cortex is crucial for scene memory (Epstein, 2008). The fast oscillation accompanied the activities in the occipital/fusiform cortex and the parahippocampal cortex (Fig. 5). The parahippocampal cortex is well known as the node for processing natural scenes (Epstein and Kanwisher, 1998; Epstein, 2008). The activities in those areas for the fast oscillation were also found in the conventional fMRI analysis (Fig. 3) and were associated with the retention interval. The visually related cortices showed activation during this interval, where there was no visual input to the participants except the fixed point. The natural scene would be retrieved as visual images during the working memory retention interval. Together with the results from the conventional fMRI analysis and the combined EEG-fMRI analysis, the visual image, which was represented by the fast oscillation, would be rehearsed at a timing of the slow oscillation in the retrosplenial cortex for the mnemonic function. This rehearsal with the interplay of slow and fast oscillations could achieve the memory retention of a natural scene.

The high spatiotemporal information from the analysis of simultaneous EEG-fMRI enables us to successfully identify the cortical activities for the scalp EEG activities. The fast oscillations accompanied the activities in the sensory cortices for the natural scene representation. The slow oscillation was tightly coupled with the activities in the mnemonic cortex. The sensory cortical activities would be rehearsed in the mnemonic circuits for memory retention. The interplay of the slow and fast oscillations could manipulate the communication of the mnemonic and sensory circuits. 


\section{REFERENCES}

Aggleton JP, Vann SD (2004) Testing the importance of the retrosplenial navigation system: lesion size but not strain matters: a reply to Harker and Whishaw. Neurosci Biobehav Rev 28:525-531.

Allen PJ, Josephs O, Turner R (2000) A method for removing imaging artifact from continuous EEG recorded during functional MRI. NeuroImage 12:230-239.

Allen PJ, Polizzi G, Krakow K, Fish DR, Lemieux L (1998) Identification of EEG events in the MR scanner: the problem of pulse artifact and a method for its subtraction. NeuroImage 8:229-239.

Axmacher N, Henseler MM, Jensen O, Weinreich I, Elger CE, Fell J (2010) Cross-frequency coupling supports multi-item working memory in the human hippocampus. Proc Natl Acad Sci U S A 107:3228-3233.

Bottini G, Cappa S, Geminiani G, Sterzi R (1990) Topographic disorientation--a case report. Neuropsychologia 28:309-312.

Buzsaki G (2004) Large-scale recording of neuronal ensembles. Nat Neurosci 7:446-451.

Canolty RT, Edwards E, Dalal SS, Soltani M, Nagarajan SS, Kirsch HE, Berger MS, Barbaro NM, Knight RT (2006) High gamma power is phase-locked to theta oscillations in human neocortex. Science 313:1626-1628.

Ekstrom AD, Kahana MJ, Caplan JB, Fields TA, Isham EA, Newman EL, Fried I (2003) Cellular networks underlying human spatial navigation. Nature 425:184-188. 
Engel AK, Singer W (2001) Temporal binding and the neural correlates of sensory awareness. Trends Cogn Sci 5:16-25.

Epstein R, Kanwisher N (1998) A cortical representation of the local visual environment. Nature 392:598-601.

Epstein RA (2008) Parahippocampal and retrosplenial contributions to human spatial navigation. Trends Cogn Sci 12:388-396.

Fries P (2005) A mechanism for cognitive dynamics: neuronal communication through neuronal coherence.

Trends Cogn Sci 9:474-480.

Fries P, Reynolds JH, Rorie AE, Desimone R (2001) Modulation of oscillatory neuronal synchronization by selective visual attention. Science 291:1560-1563.

Gratton G, Coles MG, Donchin E (1983) A new method for off-line removal of ocular artifact. Electroencephalography and clinical neurophysiology 55:468-484.

Hyvarinen A, Oja E (2000) Independent component analysis: algorithms and applications. Neural Netw $13: 411-430$.

Ino T, Inoue Y, Kage M, Hirose S, Kimura T, Fukuyama H (2002) Mental navigation in humans is processed in the anterior bank of the parieto-occipital sulcus. Neurosci Lett 322:182-186.

Jansen M, White TP, Mullinger KJ, Liddle EB, Gowland PA, Francis ST, Bowtell R, Liddle PF (2012) Motion-related artefacts in EEG predict neuronally plausible patterns of activation in fMRI data. 
NeuroImage 59:261-270.

Jensen O, Colgin LL (2007) Cross-frequency coupling between neuronal oscillations. Trends Cogn Sci $11: 267-269$.

Johnston SJ, Linden DE, Shapiro KL (2012) Functional imaging reveals working memory and attention interact to produce the attentional blink. Journal of cognitive neuroscience 24:28-38.

Kahana MJ, Sekuler R, Caplan JB, Kirschen M, Madsen JR (1999) Human theta oscillations exhibit task dependence during virtual maze navigation. Nature 399:781-784.

Kobayashi Y, Amaral DG (2000) Macaque monkey retrosplenial cortex: I. three-dimensional and cytoarchitectonic organization. J Comp Neurol 426:339-365.

Lisman JE, Idiart MA (1995) Storage of 7 +/- 2 short-term memories in oscillatory subcycles. Science 267:1512-1515.

Lisman JE, Jensen O (2013) The theta-gamma neural code. Neuron 77:1002-1016.

Maris E, Oostenveld R (2007) Nonparametric statistical testing of EEG- and MEG-data. Journal of neuroscience methods 164:177-190.

Mizuhara H, Yamaguchi Y (2011) Neuronal ensemble for visual working memory via interplay of slow and fast oscillations. Eur J Neurosci 33:1925?1934.

O'Craven KM, Kanwisher N (2000) Mental imagery of faces and places activates corresponding stiimulus-specific brain regions. Journal of cognitive neuroscience 12:1013-1023. 
O'Keefe J, Recce ML (1993) Phase relationship between hippocampal place units and the EEG theta rhythm.

Hippocampus 3:317-330.

Roux F, Wibral M, Singer W, Aru J, Uhlhaas PJ (2013) The phase of thalamic alpha activity modulates cortical gamma-band activity: evidence from resting-state MEG recordings. The Journal of neuroscience : the official journal of the Society for Neuroscience 33:17827-17835.

Scheeringa R, Fries P, Petersson KM, Oostenveld R, Grothe I, Norris DG, Hagoort P, Bastiaansen MC (2011) Neuronal Dynamics Underlying High- and Low-Frequency EEG Oscillations Contribute Independently to the Human BOLD Signal. Neuron 69:572-583.

Skaggs WE, McNaughton BL, Wilson MA, Barnes CA (1996) Theta phase precession in hippocampal neuronal populations and the compression of temporal sequences. Hippocampus 6:149-172.

Suzuki K, Yamadori A, Hayakawa Y, Fujii T (1998) Pure topographical disorientation related to dysfunction of the viewpoint dependent visual system. Cortex 34:589-599.

Tallon-Baudry C, Bertrand O, Delpuech C, Permier J (1997) Oscillatory gamma-band (30-70 Hz) activity induced by a visual search task in humans. J Neurosci 17:722-734.

Tort AB, Komorowski RW, Manns JR, Kopell NJ, Eichenbaum H (2009) Theta-gamma coupling increases during the learning of item-context associations. Proc Natl Acad Sci U S A 106:20942-20947.

Varela F, Lachaux JP, Rodriguez E, Martinerie J (2001) The brainweb: phase synchronization and large-scale integration. Nat Rev Neurosci 2:229-239. 


\section{FIGURES}

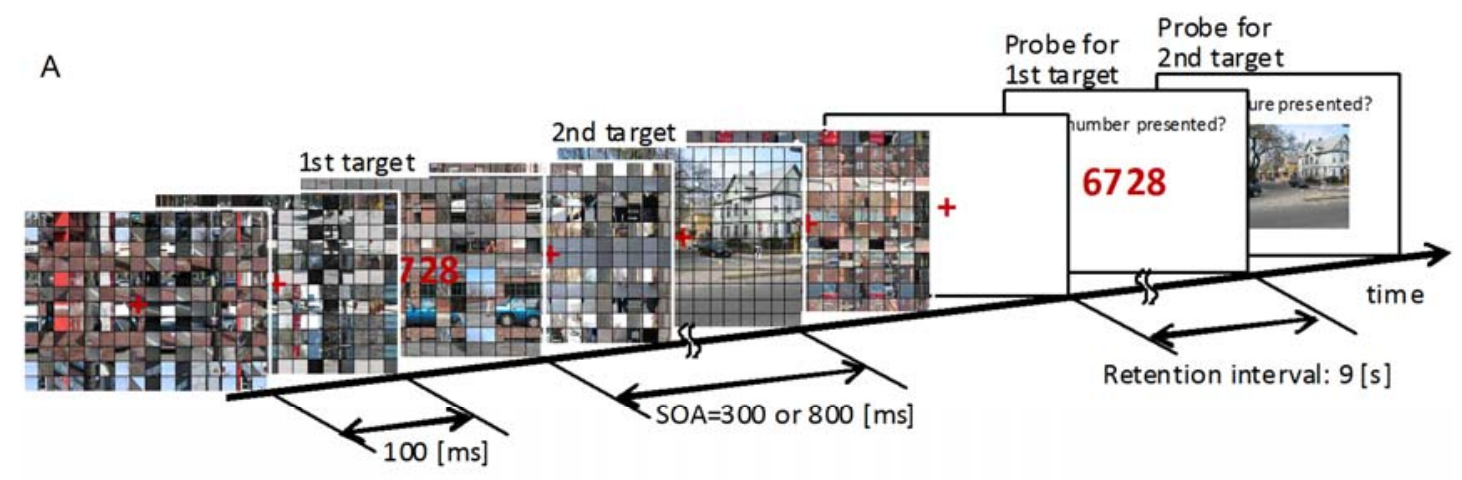

B

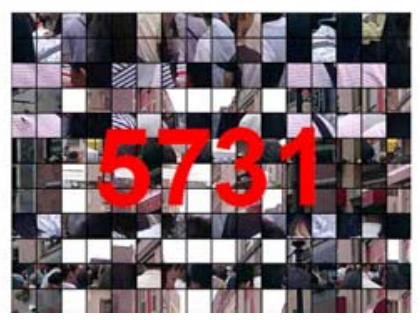

C

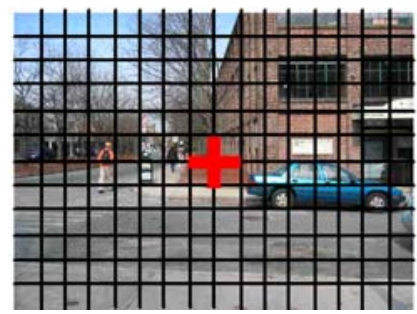

Figure 1 Temporal procedure and an example of experimental stimuli. (A) Temporal procedure of experiment. The rapid serial visual presentation (RSVP) procedure was used as the attentional blink task. Each stimulus was refreshed every $100 \mathrm{~ms}$. The SOA between the first and second targets was 300 or $800 \mathrm{~ms}$. After the $9 \mathrm{~s}$ retention interval, participants had to indicate whether the probe stimuli of both the first and second targets were present in the RSVP stream. Examples of the first target (a four-digit number) (B) and the second target (a natural scene) (C). These target stimuli embedded in a rapid serial visual presentation (RSVP) of randomly shuffled natural scene images (distractors). Two targets were presented with short SOAs (300 ms or $800 \mathrm{~ms}$ ) as an attentional blink procedure. Participants were required to retain both the first and second targets. 


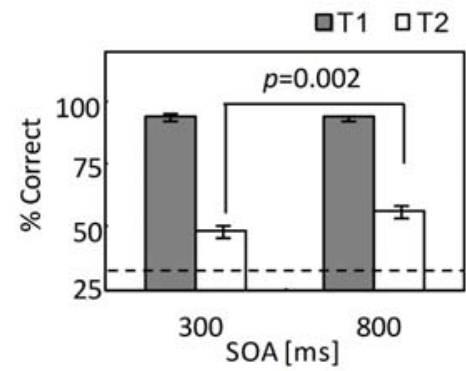

Figure 2 Behavioral results of accuracy for the recognition memory. The gray/white bars and lines represent the mean accuracies across participants and the standard errors for the first (T1) and second (T2) targets. The horizontal dashed line represents the chance rate of the accuracy. A significant difference was observed between the conditions of $\mathrm{SOA}=300 \mathrm{~ms}$ and $\mathrm{SOA}=800 \mathrm{~ms}$ for the second target retention $(\mathrm{n}=14)$. 


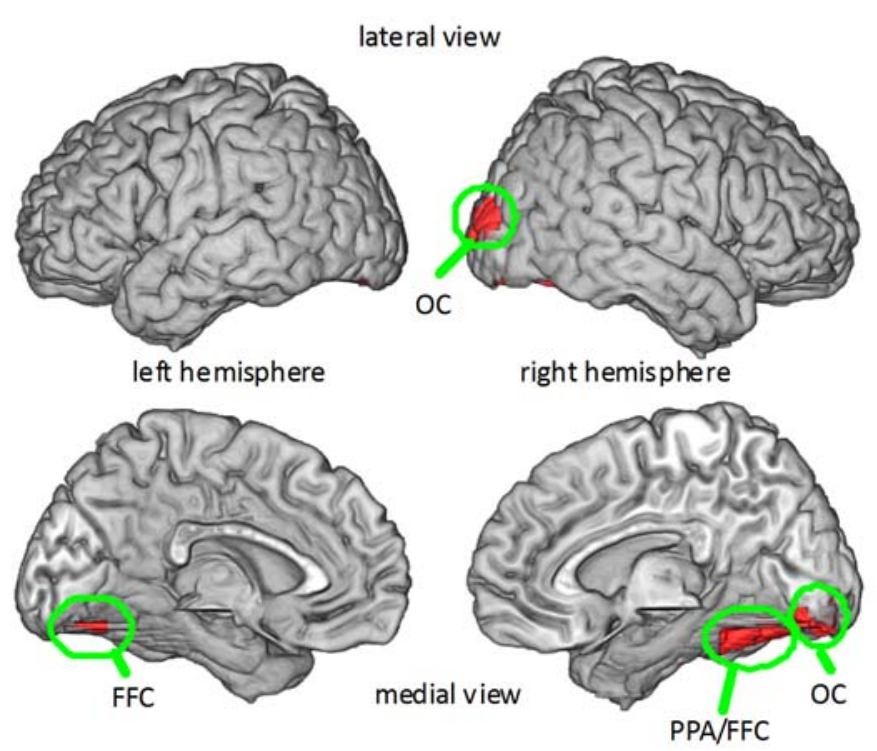

Figure 3 Results of fMRI activations for working memory retention. The activations were identified by comparing the conditions of $\mathrm{SOA}=300 \mathrm{~ms}$ and $\mathrm{SOA}=800 \mathrm{~ms}$. The red blobs represent the larger activity in the condition of $\mathrm{SOA}=800 \mathrm{~ms}$ relative to $\mathrm{SOA}=300 \mathrm{~ms}$ ( $\mathrm{n}=14$, cluster-level FDR corrected $\mathrm{p}<0.05$ ). OC: occipital cortex, FFC: fusiform cortex, PPA: parahippocampal place area. 
A
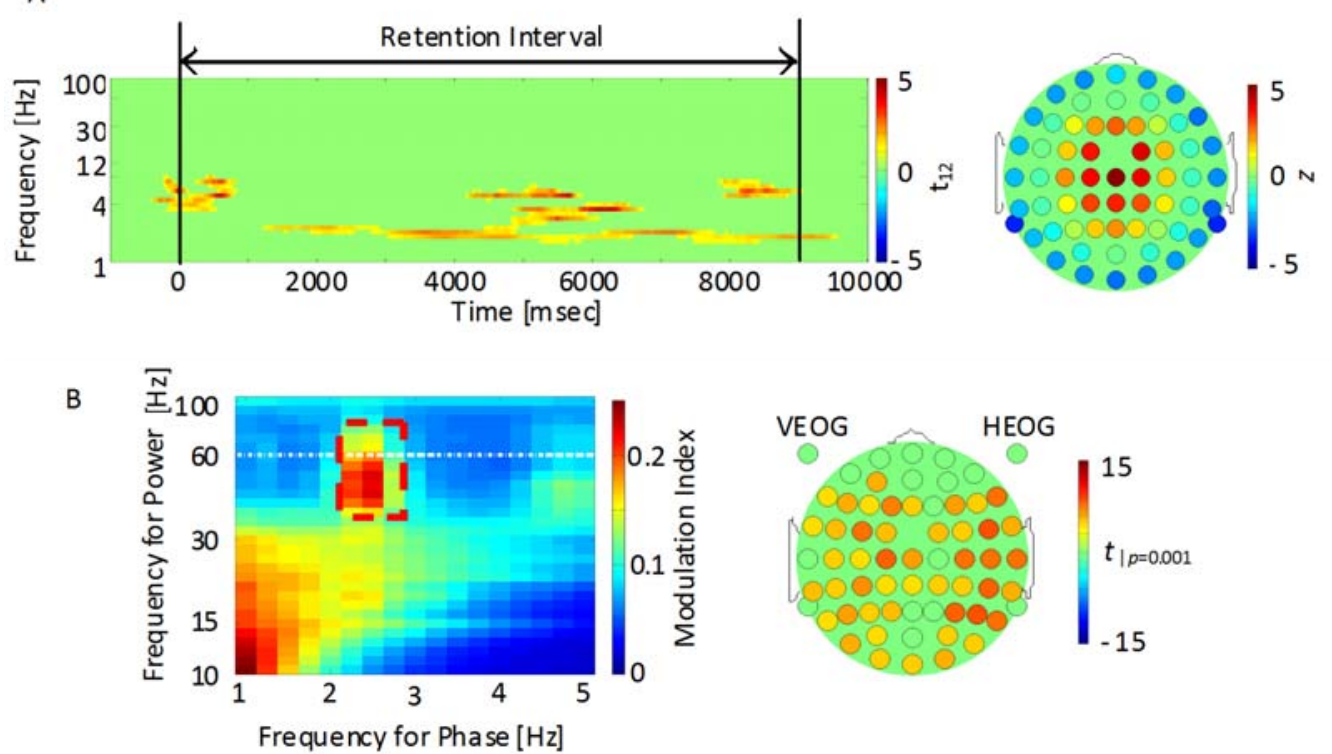

Figure 4 Results of EEG analysis. (A) Results of EEG power modulation associated with working memory retention (corrected $\mathrm{p}<0.05$ ). The EEG power derived from the ICA components was compared between the conditions of $\mathrm{SOA}=800 \mathrm{~ms}$ and $\mathrm{SOA}=300 \mathrm{~ms}(\mathrm{n}=13)$. The warm color represents the EEG power enhancement in the condition of $\mathrm{SOA}=800 \mathrm{~ms}$ relative to the condition of $\mathrm{SOA}=300 \mathrm{~ms}$. The topography was derived from the averaging of the z-scored channel weight of ICA decomposition by demeaning and dividing by the SD. (B) Results of the phase-amplitude coupling between the slow $(1-5 \mathrm{~Hz})$ and fast $(10-100 \mathrm{~Hz}$ in logarithm step) oscillations. The phase-amplitude map was obtained by averaging the modulation index over the EEG electrodes for the condition of $\mathrm{SOA}=800 \mathrm{~ms}$. The notch filter frequency is represented as the white dashed line. The topography represents the distribution of the phase-amplitude coupling strength by comparing the modulation index shown by the red square between the condition of SOA= $800 \mathrm{~ms}$ and the surrogate data (corrected $\mathrm{p}<0.001$ ). 


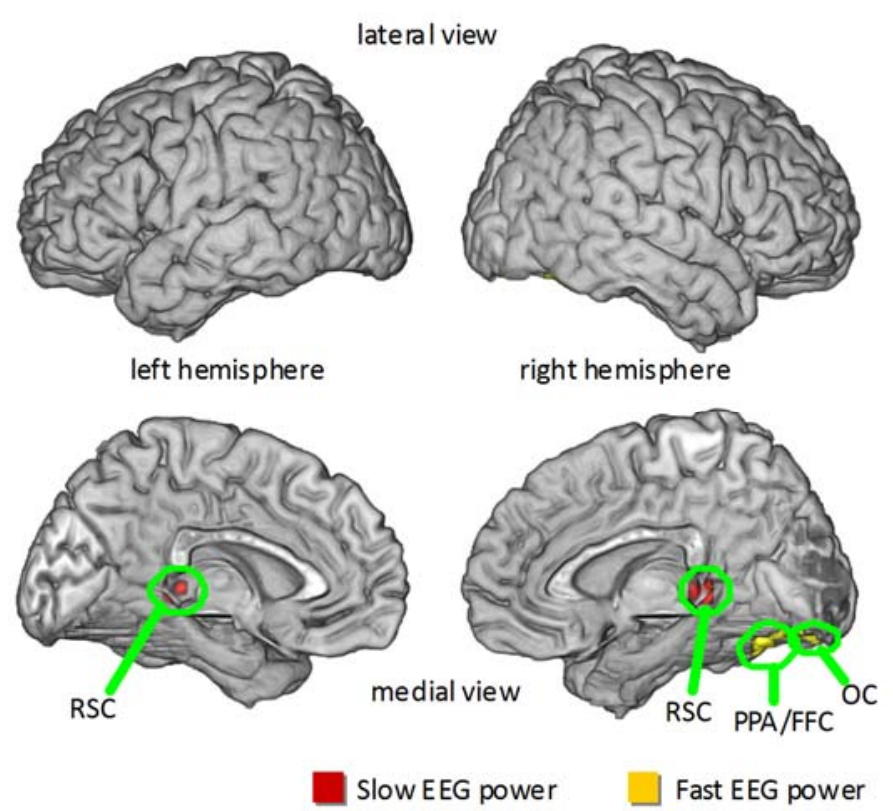

Figure 5 Results of fMRI activities in association with the slow and fast EEG power modulation. The activities were identified by computing the correlation of the fMRI-BOLD and EEG power time series. The red blobs represent the positive correlation to the slow EEG power (1-4 Hz), and yellow blobs represent the positive correlation to the fast EEG power $(30-100 \mathrm{~Hz}$ ), respectively $(\mathrm{n}=13$, cluster-level FDR corrected $\mathrm{p}<0.05)$. OC: occipital cortex, FFC: fusiform cortex, PPA: parahippocampal place area, RSC: retrosplenial cortex. 


\section{TABLES}

Table 1 The fMRI results of anatomical regions, seed voxel coordinates (MNI), and t-value for the significant activations in association with successful memory retention ( $\mathrm{SOA}=800 \mathrm{~ms}>\mathrm{SOA}=300 \mathrm{~ms}$ ).

\begin{tabular}{lccccc}
\hline \hline Anatomical region & BA & \multicolumn{3}{c}{ MNI coordinates (mm) } & t-value \\
\cline { 2 - 5 } & & $\mathrm{x}$ & $\mathrm{y}$ & $\mathrm{z}$ & \\
\hline \hline R Parahippocampal place area/Fusiform cortex & $37 / 18$ & 28 & -63 & -2 & 5.32 \\
R Occipital cortex & 19 & 28 & -85 & 16 & 5.58 \\
L Fusiform cortex & 18 & -24 & -82 & -5 & 4.11 \\
\hline Results are thresholded at $\mathrm{p}<0.005$ (uncorrected for the multiple comparison) and an extent \\
threshold of 28 voxels, resulting in $\mathrm{p}<0.05$ (cluster-level FDR corrected). BA: Brodmann area, MNI: \\
Montreal Neurological Institute.
\end{tabular}

Table 2 fMRI results of anatomical regions, seed voxel coordinates (MNI), and t-value for the significant activations in association with slow and fast EEG power modulation.

\begin{tabular}{|c|c|c|c|c|c|}
\hline \multirow[t]{2}{*}{ Anatomical region } & \multirow[t]{2}{*}{ BA } & \multicolumn{3}{|c|}{ MNI coordinates (mm) } & \multirow[t]{2}{*}{ t-value } \\
\hline & & $\mathrm{x}$ & $\mathrm{y}$ & $\mathrm{z}$ & \\
\hline \multicolumn{6}{|l|}{ Slow EEG power } \\
\hline \multicolumn{6}{|l|}{ Positive Correlation: } \\
\hline Retrosplenial cortex & 27 & -7 & -36 & 7 & 5.41 \\
\hline L Parahippocampal place area/Fusiform cortex & $37 / 18$ & 36 & -64 & -12 & 3.89 \\
\hline L Occipital cortex & 19 & 29 & -83 & -9 & 4.24 \\
\hline L/R Supplementary motor cortex & 6 & 0 & -7 & 61 & 4.08 \\
\hline \multicolumn{6}{|l|}{ Negative Correlation: } \\
\hline \multicolumn{6}{|c|}{ None } \\
\hline \multicolumn{6}{|l|}{ Fast EEG power } \\
\hline \multicolumn{6}{|l|}{ Positive Correlation: } \\
\hline L Parahippocampal place area/Fusiform cortex & $37 / 18$ & 36 & -61 & -12 & 4.13 \\
\hline L Occipital cortex & 19 & 29 & -83 & -9 & 4.24 \\
\hline \multicolumn{6}{|l|}{ Negative Correlation: } \\
\hline \multicolumn{6}{|c|}{ None } \\
\hline $\begin{array}{l}\text { Results are thresholded at } \mathrm{p}<0.005 \text { (uncor } \\
\text { threshold of } 19 \text { voxels for slow oscillation } \\
\text { (cluster-level FDR corrected). BA: Brodmann a }\end{array}$ & $\begin{array}{l}\text { cted for } \\
\text { d } 36 \text { vos } \\
\text { a, MNI: } \Lambda\end{array}$ & for & $\begin{array}{l}\text { com } \\
\text { scilla } \\
\text { blogic }\end{array}$ & $\begin{array}{l}\text { n) an } \\
\text { esultin } \\
\text { tute. }\end{array}$ & $\begin{array}{l}\text { extent } \\
p<0.05\end{array}$ \\
\hline
\end{tabular}

